\title{
Anti-CD20-CAR-CD3zeta-4-1BB-expressing Autologous T-lymphocyte Cells
}

National Cancer Institute

\section{Source}

National Cancer Institute. Anti-CD20-CAR-CD3zeta-4-1BB-expressing Autologous Tlymphocyte Cells. NCI Thesaurus. Code C104006.

A preparation of autologous blood T-lymphocytes that have been genetically modified to express a chimeric antigen receptor (CAR) consisting of an anti-CD20 scFv (single chain variable fragment); the cytoplasmic portion of the human TCR-[zeta] molecule; and the co-stimulatory molecule 4-1BB (CD137), with potential immunostimulating and antineoplastic activities. Upon transfusion, anti-CD20-CAR-CD3zeta-4-1BB-expressing autologous T-lymphocyte cells direct T-cells to CD20-expressing tumor cells. This results in cytotoxic T lymphocyte (CTL) and antibody responses against CD20-expressing tumor cells, causing tumor cell lysis. The CD20 antigen, a non-glycosylated cell surface phosphoprotein, is a B-cell specific cell surface antigen expressed in B-cell lineage malignancies. CD3 zeta is one of several membrane-bound polypeptides found in the Tcell receptor (TCR)/CD3 complex and regulates the assembly of complete TCR complexes and their expression on the cell surface. The 4-1BB co-stimulatory molecule signaling domain enhances activation and signaling after recognition of CD20; the inclusion of this signaling domain may increase the antitumor activity compared to the inclusion of the CD3-zeta chain alone. 